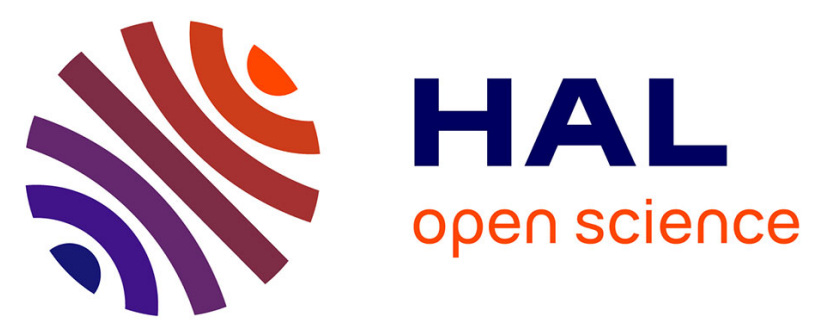

\title{
MYPT1, the targeting subunit of smooth muscle myosin phosphatase, is a substrate for the asparaginyl hydroxylase factor inhibiting hypoxia inducible factor (FIH)
}

James D Webb, Andrea Murányi, Christopher W Pugh, Peter J Ratcliffe, Mathew L Coleman

\section{To cite this version:}

James D Webb, Andrea Murányi, Christopher W Pugh, Peter J Ratcliffe, Mathew L Coleman. MYPT1, the targeting subunit of smooth muscle myosin phosphatase, is a substrate for the asparaginyl hydroxylase factor inhibiting hypoxia inducible factor (FIH). Biochemical Journal, 2009, 420 (2), pp.327-333. 10.1042/BJ20081905 . hal-00479098

\section{HAL Id: hal-00479098 \\ https://hal.science/hal-00479098}

Submitted on 30 Apr 2010

HAL is a multi-disciplinary open access archive for the deposit and dissemination of scientific research documents, whether they are published or not. The documents may come from teaching and research institutions in France or abroad, or from public or private research centers.
L'archive ouverte pluridisciplinaire $\mathbf{H A L}$, est destinée au dépôt et à la diffusion de documents scientifiques de niveau recherche, publiés ou non, émanant des établissements d'enseignement et de recherche français ou étrangers, des laboratoires publics ou privés. 
MYPT1, the targeting subunit of smooth muscle myosin phosphatase, is a substrate for the asparaginyl hydroxylase Factor Inhibiting Hypoxia Inducible Factor (FIH).

James D Webb*, Andrea Murányi ${ }^{\dagger}$, Christopher W Pugh*, Peter J Ratcliffe* and Mathew L Coleman*

*Henry Wellcome Building for Molecular Physiology, Nuffield Department of Clinical Medicine, University of Oxford, Roosevelt Drive, Headington, OXFORD OX3 7BN, UK

${ }^{\dagger}$ Department of Nutritional Sciences, University of Arizona, Shantz Building, Room 309, 1177 East Fourth Street, Tucson, AZ 85721-0038, USA

Corresponding author:

Mathew L Coleman

mcoleman@well.ox.ac.uk

Tel. $\quad+44(0) 1865287807$

Fax $\quad+44(0) 1865287787$ 


\begin{abstract}
The asparaginyl hydroxylase $\mathrm{FIH}$ was first identified as a protein that inhibits transcriptional activation by hypoxia inducible factor (HIF), through hydroxylation of an Asn residue in the Cterminal activation domain (HIF-CAD). More recently, several Ankyrin Repeat Domain (ARD) containing proteins were identified as $\mathrm{FIH}$ substrates using $\mathrm{FIH}$ interaction assays. Although the function(s) of these ARD hydroxylations is unclear, expression of the ARD protein Notch 1 was shown to compete efficiently with HIF-CAD for Asn hydroxylation, and thus to enhance HIF activity. The ARD is a common protein domain with over 300 examples in the human proteome. However, the extent of hydroxylation among ARD proteins, and the ability of other members to compete with HIF-CAD for FIH, is not known. Here we assay for Asnhydroxylation in a bioinformatically predicted FIH substrate, the targeting subunit of myosin phosphatase, MYPT1. Our results confirm hydroxylation both in cultured cells and in endogenous protein purified from animal tissue. We show that the extent of hydroxylation at three sites is dependent on FIH expression level, and that hydroxylation is incomplete under basal conditions even in the animal tissue. We also show that expression of MYPT1 enhances HIF-CAD activity in a manner consistent with competition for $\mathrm{FIH}$, and that this property extends to other ARD proteins. These results extend the range of FIH substrates, and suggest that cross-competition between ARDs and HIF-CAD, and between ARDs themselves, may be extensive and have important effects on hypoxia signalling.
\end{abstract}

\title{
Running title
}

MYPT1 is a substrate for FIH

\section{Keywords}

Hydroxylation

Myosin phosphatase

Hypoxia Inducible Factor

Ankyrin Repeat Domain

Factor Inhibiting HIF

\section{Abbreviations}

Hypoxia Inducible Factor, HIF

Factor Inhibiting Hypoxia Inducible Factor, FIH

Myosin Phosphatase Targeting Subunit 1, MYPT1

Ankyrin Repeat, AR

Ankyrin Repeat Domain, ARD

C-terminal transactivation domain, $C A D$

Inhibitor of NfKappaB alpha, $1 \kappa B \alpha$

Ankyrin Repeat and SOCS Box protein 4, ASB4

Intracellular domain, ICD

Myosin Light Chain, MLC

Protein Phosphatase type 1, PP1

Liquid Chromatography, LC

Tandem Mass Spectrometry, MS/MS 


\section{Introduction}

Factor inhibiting hypoxia inducible factor $(\mathrm{FIH})$ is an asparaginyl hydroxylase that contributes to oxygen-dependent regulation of hypoxically-induced genes by hydroxylation of Hypoxia Inducible transcription Factor (HIF) [1, 2]. Hydroxylation of the HIF C-terminal transactivation domain (CAD) suppresses HIF activity by preventing recruitment of the p300/CBP transcriptional co-activators to HIF target genes [3, 4].

Recently several novel substrates of FIH have been identified that are targeted for Asn hydroxylation within a protein:protein interaction motif known as the Ankyrin Repeat (AR) Domain (ARD). These experiments have demonstrated that Asn hydroxylation occurs at multiple sites within the ARDs of several proteins, including; $I_{\kappa} B \alpha$, Notch1 - 3, ASB4, Rabankyrin and RNaseL [5-8]. Quantitative MS/MS analyses have demonstrated that the extent of hydroxylation may vary between sites in the same ARD, and is generally incomplete at any given site, at least in cells grown in the standard tissue culture conditions used in these experiments. However, despite the identification of multiple ARD substrates of $\mathrm{FIH}$, functional insights into the role of ARD hydroxylation have so far been limited $[5,6]$. Though effects of $\mathrm{FIH}$ have been reported on biological functions of some of these target proteins it is not yet clear how they relate to hydroxylation $[7,9]$.

ARDs contain variable numbers of ARs which consist of paired anti-parallel $\alpha$-helices that stack upon one another to form a protein interaction surface [10]. Although hydroxylation of the Notch1 ARD promotes the formation of an intra-repeat hydrogen bond postulated to enhance structural stability, it does not alter the classical ARD conformation [6]. The clearest biological action of an FIH-mediated ARD hydroxylation defined to date is the crosscompetition between Notch 1 and HIF-CAD for Asn hydroxylation. Thus expression of the ARD of Notch1 competitively inhibits FIH-mediated HIF-CAD Asn hydroxylation, resulting in enhanced HIF-CAD activity [6].

Although yeast 2-hybrid and proteomic screens have identified only a relatively small number of ARD substrates of FIH [5, 6] the human genome encodes over 300 ARD proteins, most of which contain consensus motifs for FIH-mediated hydroxylation (SMART database [11]). This raises important questions as to whether hydroxylation occurs on other ARD proteins and if so, to what extent, what roles hydroxylation might play in hypoxia signalling, and what the implications are for cross-competition with HIF for Asn hydroxylation.

To address these questions, and to determine whether Asn hydroxylation might occur on ARD proteins not captured in previous screens, we chose to study a bioinformaticallyidentified candidate substrate, myosin phosphatase targeting subunit 1 (MYPT1). MYPT1 is an ARD-containing protein with established functions in contractile regulation that are of potential interest to hypoxia biology [12], and for which there is structural information that might guide analysis of functional effects [13].

We show that the ARD of MYPT1 contains three sites of asparaginyl hydroxylation, and that these hydroxylations are $\mathrm{FIH}$-dependent. For the first time we show that Asn hydroxylation occurs in an ARD protein purified from an animal: this hydroxylation is incomplete, suggesting that previous results from tissue culture cells give a true reflection of ARD hydroxylation in vivo. Further, we show that MYPT1, and other ARD substrates of $\mathrm{FIH}$, can modulate the transcriptional activity of HIF-CAD through competitive FIH inhibition. 


\section{Materials and methods}

\section{Cell culture}

293T, HeLa and MCF7 cells were maintained in Dulbecco's modified Eagle's medium supplemented with $10 \%(\mathrm{v} / \mathrm{v})$ foetal calf serum, $2 \mathrm{mM}$ L-glutamine, 50 IU (international units) $\mathrm{ml}^{-1}$ penicillin and $50 \mathrm{\mu g} \mathrm{ml}^{-1}$ streptomycin.

\section{Plasmids}

Human MYPT1 ARD (residues 1 - 296) was amplified by PCR and cloned into pEF1/V5-His A (Invitrogen). Wild-type FIH and D201A FIH plasmids were as described previously [6]. For reporter gene assays, full-length human MYPT1, human MYPT1 ARD (residues 1 - 296), mouse Notch1 intracellular domain (ICD, residues 1751 - 2531), full-length human Tankyrase1, full-length human $\mathrm{p} 105$, full-length human $\mathrm{I}_{\kappa} \mathrm{B} \alpha$, full-length human Rabankyrin5 and full-length human RNaseL were amplified by PCR and cloned into p3xFLAG-CMV-10 (Sigma). The integrity of all constructs was verified by DNA sequence determination.

\section{Transfection and reporter gene assays}

Cells were transfected in dishes of $10 \mathrm{~cm}$ or $15 \mathrm{~cm}$ diameter, using $4.5 \mu \mathrm{g}$ or $10 \mu \mathrm{g}$ total plasmid DNA respectively and FuGENE® 6 transfection reagent (Roche Applied Science) according to the manufacturer's instructions. Where applicable, MYPT1, ARD, and FIH expression plasmids were co-transfected in a $4: 1$ ratio. The transcriptional activity of HIF $1 \alpha$ CAD was analysed in HeLa cells using a Gal4DBD-HIF-CAD fusion protein and UASluciferase reporter system as described previously [6].

\section{Knock-down of FIH expression using siRNA}

Human FIH (target F1) and control Drosophila HIF (dHIF) duplexes have been described previously [14], and were synthesized by Ambion. Cells were transfected twice at $24 \mathrm{~h}$ intervals using a $20 \mathrm{nM}$ dose of duplex and Oligofectamine reagent (Invitrogen), according to the manufacturer's instructions.

\section{Antibodies}

Anti-FIH antibody was as described previously [14]. Anti-MLC (M4401), anti- $\beta$-tubulin (T5293) and anti-FLAG M2 peroxidase (A8592) antibodies were from Sigma. Anti-phospho-MLC antibodies (Ser-19 and Ser-19/Thr-18) were from Cell Signaling (3671 and 3674 respectively). Anti-PPIc $\delta$ was from Abcam (ab53315). Anti-MYPT1 antibody, F38, was as described [15].

\section{Immunoprecipitation and immunoblotting}

Cells were lysed in Jie's buffer [ $\mathrm{NaCl} 100 \mathrm{mM}$, TrisHCl pH $7.520 \mathrm{mM}, \mathrm{MgCl}_{2} 5 \mathrm{mM}$, Nonidet P-40 0.5\% (v/v)] supplemented with Complete, EDTA-free protease inhibitor cocktail (Roche Applied Science). V5-tagged MYPT1 ARD was immunoprecipitated with anti-V5 agarose affinity gel (Sigma). Endogenous MYPT1 was immunoprecipitated by pre-incubation with antiMYPT1 antibody followed by incubation with protein A agarose (Upstate Biotechnology). For immunoblotting of phospho-MLC, cells were lysed directly into 3x SDS sample buffer and sonicated briefly. Lysates or immunoprecipitates were resolved by SDS-PAGE, electroblotted onto PVDF membranes (Millipore) and probed using the indicated primary antibodies. Horseradish peroxidase-conjugated anti-mouse or anti-rabbit secondary antibodies (Dako) were used with either SuperSignal West Pico or SuperSignal West Dura (Pierce Biotechnology) to visualize immunoreactive species. Where necessary when immunoblotting immunoprecipitates, anti-rabbit TrueBlot horseradish peroxidase-conjugated secondary antibody (eBioscience) was used to avoid signal from immunoprecipitating immunoglobulin.

Protein digestion and liquid chromatography-MS analysis

After resolution by SDS-PAGE and staining, protein-containing bands were excised and digested in bicarbonate buffer using either sequencing grade trypsin (Promega) or sequencing grade endoproteinase GluC (Roche Applied Science) as described [16]. Liquid chromatography was performed using an Ultimate system equipped with a Famos autosampler and C18 PepMap column (LC Packings). The chromatography system was connected directly to a UV flow cell (Ultimate) and a 3D high-capacity ion trap mass spectrometer (HCTplus, Bruker Daltonics) via a pneumatically assisted nano-electrospray source. Silica-coated Picotips (New Objective) were used for electrospray ionization. 
Instruments were controlled using HyStar 3.0 and EsquireControl 5.2 software (Bruker Daltonics). Raw chromatography data were processed and Mascot-compatible files generated using DataAnalysis 3.2 software (Bruker Daltonics). Searches were performed using Mascot software (Matrix Science [17]) using the most recent releases of the SwissProt or NBCInr databases, following which MS/MS spectra and extracted ion chromatograms were analysed in detail using DataAnalysis 3.2.

\section{Protein purification}

Endogenous myosin phosphatase holoenzyme was purified from turkey gizzard as described [18].

\section{Statistical analysis}

Unless otherwise stated quantitative data are represented as the mean $\pm S$. E. M. of three experiments. Statistical significance was evaluated using Student's $t$ test. 


\section{Results}

MYPT1 is a potential FIH substrate

FIH-dependent Asn hydroxylation sites within ARDs conform to a $\mathrm{L}_{(-8)} \mathrm{D} / \mathrm{E}_{(-2)} \mathrm{XN}$ motif specifically located to the $\beta$ hairpin loops that connect ARs [5-7]. Bioinformatic alignment with known FIH substrates (Figure 1) indicated that the targeting subunit of smooth muscle myosin phosphatase, MYPT1, contains two potential hydroxylation sites within the $\beta$-hairpin loops of its $A R D$ that match the $\mathrm{L}_{(-8)} \mathrm{D} / \mathrm{E}_{(-2)} \mathrm{XN}$ consensus (Asn-67 and Asn-226) and a further two conforming to $L_{(-8)} N$ (Asn-100 and Asn-288). Three of these sites (Asn-67, Asn-100 and Asn226) have additional similarities to previously identified substrates in the primary sequence (Figure 1).

The ARD of MYPT1 is hydroxylated in vivo

To determine if any of the identified Asn residues were hydroxylated in vivo we immunopurified V5-tagged human MYPT1 ARD from transfected 293T cells. The purified ARD protein was resolved by SDS-PAGE and digested using either trypsin or endoproteinase GluC before analysis by liquid chromatography-tandem mass spectrometry (LC-MS/MS). Peptides containing all four candidate asparagine residues were observed, and MS/MS sequencing assigned hydroxylation at Asn-67, Asn-100 and Asn-226 (Figures 2 and S1). No hydroxylation was detected at Asn-288 (data not shown), the site with the least similarity to known FIH substrates (Figure 1).

FIH is necessary and sufficient for hydroxylation of the MYPT1 ARD

In order to determine whether MYPT1 was a substrate for $\mathrm{FIH}$ we quantified hydroxylation at Asn-67, Asn-100 and Asn-226 by liquid chromatography-mass spectrometry (LC-MS) under conditions of various FIH interventions. 293T cells were transfected with small interfering RNA (siRNA) duplexes targeting FIH or a control sequence (dHIF), then subsequently transfected with MYPT1 ARD plus pcDNA3 vector (EV), FIH or a hydroxylation-defective FIH mutant (D201A). After immunoprecipitation and digestion, the extent of hydroxylation at each site was determined by LC-MS. In the presence of endogenous levels of FIH each of the three sites was around $5-10 \%$ hydroxylated at the target Asn (Figure 3A). Overexpression of FIH increased the level of hydroxylation to approximately $90 \%$ whilst overexpression of the FIH D201A mutant had no effect. Suppression of FIH by siRNA reduced the extent of hydroxylation at all three sites. Although consistent across the three sites this effect of FIH suppression was not statistically significant. This is likely to reflect the low basal levels of MYPT1 hydroxylation present under the experimental conditions, which were approaching the limits of detection by mass spectrometry, combined with the incomplete siRNA-mediated suppression of FIH (Figure 3B).

These results thus confirm bioinformatic predictions that MYPT1 contains multiple sites of FIH-dependent hydroxylation within its ARD, consistent with the hypothesis that FIH-mediated hydroxylation is a general phenomenon among ARD-containing proteins. They also indicate that, at least in transfected tissue culture cells, hydroxylation of individual ARs is incomplete.

\section{Endogenous MYPT1 is hydroxylated}

To test whether Asn hydroxylation occurs in the full length protein in vivo, and to assess the extent of hydroxylation in normal tissue, we next sought to determine whether a sample of endogenous animal MYPT1 was hydroxylated. A sample of myosin phosphatase holoenzyme, purified from turkey gizzard, was resolved by SDS-PAGE and stained with Coomassie blue. The MYPT1 species was excised and digested with trypsin or GluC, then analysed by LC-MS/MS and LC-MS.

Although the turkey MYPT1 gene has not been sequenced, the amino acid sequence of the chicken protein is available (NCBInr database, gi number 633038). As conservation of the MYPT1 ARD between human and chicken is greater than $96 \%$, it seems reasonable to assume a high degree of conservation between the chicken and turkey sequences. Searching the MS/MS fragmentation data against the chicken protein sequence correctly identified many predicted peptides, validating this assumption. MS/MS sequencing confirmed hydroxylation at Asn-67 and Asn-100 (Figure S2), and LC-MS quantified the degree of hydroxylation at the Asn-67 and Asn-100 sites as 48\% and 43\%, respectively (Figure 4). A peptide containing 
Asn-288 was observed by GluC digestion, but in agreement with the data from cell culture experiments no hydroxylation was detected at this site (data not shown). A peptide containing Asn-226 was not detected in these experiments, likely due to a Gly to Arg substitution four residues N-terminal to Asn-226 in the chicken protein relative to human MYPT1, which results in a short tryptic fragment that is not amenable to detection by MS.

FIH does not affect the interaction between MYPT1 and PPIC $\delta$ nor MLC phosphorylation We next sought to understand what the consequences of Asn-hydroxylation are on the function of myosin phosphatase. MYPT1 regulates myosin light chain (MLC) phosphorylation by recruiting the protein phosphatase type 1c $\delta$ isoform (PP1c $\delta$ ), by imparting selectivity towards MLC and by fully activating the myosin phosphatase complex [19].

The structure of the N-terminus of MYPT1 in complex with PP1c $\delta$ shows that the main binding site for PP1c $\delta$ is the well-characterized PP1 binding motif (KVKF) located immediately Nterminal to AR1 [13]. Additional interactions are mediated by the ARD: the $\beta$-hairpin loops of the two groups of ARs form a 'clamp' that stabilizes the C-terminal tail of PP1c $\delta$ (Figure S3). Several residues in the MYPT1 ARD interact with PP1c $\delta$, including Val-71 which is adjacent to Asn-67, and Asp-229 and Trp-231 which are C-terminal to Asn-226: this raises the possibility that hydroxylation of the Asn residues could modulate the interaction with PP1c . To test this we immunoprecipitated endogenous MYPT1 from 293T cells transfected with pcDNA3 vector (EV), FIH, D201A FIH, control siRNA (dHIF) or FIH siRNA. Small amounts of overexpressed FIH were observed co-immunoprecipitating with endogenous MYPT1. However, neither $\mathrm{FIH}$ overexpression nor knock-down affected the interaction between MYPT1 and PP1c $\delta$ (Figure 5A).

Structural data indicate that binding of phosphorylated MLC to the myosin phosphatase holoenzyme is mediated by an acidic groove created by the catalytic cleft of PP1c $\delta$ and the $\alpha$ helices of the MYPT1 ARs [13]. It is thought that this interaction promotes the activity and specificity of PP1c $\delta$ for phosphorylated MLC. To determine whether FIH-mediated MYPT1 ARD hydroxylation regulates PP1c $\delta$ activity towards phosphorylated MLC we asked whether FIH overexpression or knock-down had any effect on MLC phosphorylation using antibodies specific for phosphorylated MLC (Ser-19 or Thr-18/Ser-19). FIH knock-down or overexpression did not alter the levels of phosphorylated MLC in 293T, HeLa or MCF7 cells, nor did they significantly affect the level of MYPT1 (Figure 5B).

Therefore, FIH-mediated Asn hydroxylation does not appear to affect the function of myosin phosphatase dramatically, at least under the conditions tested.

MYPT1 and other ARD-containing proteins can influence HIF transcriptional activity through competition for FIH

We have previously shown that Notch1 overexpression competitively inhibits HIF-CAD hydroxylation [6]. This competition results in enhanced HIF-CAD activity that can be detected in a luciferase reporter assay based on UAS Luciferase and a HIF-CAD-GAL4 DNA binding domain (GAL4 DBD) fusion. To test whether MYPT1 also enhances HIF-CAD activity, and whether this property is observed more generally among ARD substrates of $F I H$, HeLa cells were transfected with HIF-CAD:GAL4 DBD, UAS luciferase and 3xFLAG-tagged ARDcontaining proteins. As expected, extracts from cells transfected with Notch1 ICD displayed luciferase activity five to six-fold higher than control samples expressing only HIF-CAD:GAL4 DBD and UAS Luciferase, reflecting reduction in FIH-mediated suppression of HIF-CAD activity by Notch1 (Figure 6A). Consistent with competitive inhibition of HIF-CAD hydroxylation, full-length MYPT1 and MYPT1 ARD also greatly enhanced HIF-CAD activity (six to seven-fold increase in luciferase activity), with a smaller effect (three-fold increase in luciferase activity) observed for RNaseL. Tankyrase1 (a novel ARD FIH substrate - Matthew E Cockman, personal communication) and $I \kappa B \alpha$ showed a small but significant effect on HIFCAD activity in this assay whereas p105 and Rabankyrin5 did not. When expressed at maximal plasmid doses, Rabankyrin5 and $I \kappa B \alpha$ elicited much greater fold increases in luciferase activity, in contrast to p105 and Tankyrase1 which did not (data not shown). These data therefore suggest that many, but not all, ARD FIH substrates have the potential to inhibit 
B Biochemical Journal Immediate Publication. Published on 27 Feb 2009 as manuscript BJ20081905

FIH-dependent suppression of HIF-CAD activity and that this competitive effect varies between ARD proteins. 


\section{Discussion}

We show here that the targeting subunit of the smooth muscle myosin phosphatase holoenzyme, MYPT1, is a novel FIH substrate and that its ARD is targeted for Asn hydroxylation at multiple independent sites. The initial candidacy of these sites was based on bioinformatic alignment with known FIH substrates. Similar analysis suggests that most ARD proteins contain at least one candidate FIH hydroxylation site that fulfils the dual requirements of conforming to the consensus and being located on the $\beta$-hairpin loop of the AR. We therefore infer that the hydroxylation of ARDs by FIH is likely to be common, though perhaps not universal.

Of the four predicted hydroxylation sites in the eight ARs of the MYPT1 ARD, only Asn-288 was shown not to be an FIH substrate. It is unclear at present whether this is due to the divergent sequence surrounding Asn-288 relative to other FIH substrates (Figure 1), or to the fact that AR8 does not adopt a canonical AR fold [13]. Interestingly, Asn-100 was hydroxylated at a similar level to Asn-67 and Asn-226, despite the absence of D/E in the previously described $\mathrm{L}_{(-8)} \mathrm{D} / \mathrm{E}_{(-2)} \mathrm{XN}$ motif, showing that an acidic residue is not essential for hydroxylation at this position in all ARD substrates. Taken together the results presented here provide some insight into the primary sequence determinants that regulate $\mathrm{FIH}$-mediated ARD hydroxylation, and suggest that structural factors may also contribute.

Despite the proximity of known interaction sites between MYPT1 and PP1c $\delta$ to two of the three Asn hydroxylation sites characterized here, we did not observe any significant effect of FIH manipulation on the MYPT1:PP1c $\delta$ interaction, nor on the activity of myosin phosphatase towards MLC. With respect to the MYPT1:PP1c $\delta$ interaction this may be unsurprising since the high affinity binding site is located N-terminal to the ARD [20-22]. Furthermore, Asn-67 and Asn-226 project away from PP1c $\delta$ (Figure 5A) and may therefore not be expected to modify the described interactions, despite their close proximity [13]. Likewise, the $\beta$-hairpin loops, which contain the hydroxylated Asn residues, are located on the opposite face of the ARD from that which contributes to the acidic groove of the MLC binding site, consistent with the absence of detectable regulation of MLC phosphorylation by FIH. Although the lack of effect of Asn hydroxylation on MYPT1 signalling is consistent with similar findings for other ARD substrates, including $I \kappa B \alpha$ and Notch $[5,6]$, it is possible that FIH regulates myosin phosphatase under other specific conditions.

One physical consequence of Notch1 ARD Asn hydroxylation is the formation of a hydrogen bond between the newly-created hydroxyl group and an acceptor residue two positions $\mathrm{N}$ terminal to the target Asn [6]. In all previously characterized FIH substrates the predicted acceptor group is the carboxylate of an Asp residue [5-7]. However, most candidate FIH sites without the $\mathrm{D} / \mathrm{E}$ of the $\mathrm{L}_{(-8)} \mathrm{D}_{/ \mathrm{E}_{(-2)}} \mathrm{XN}$ motif still contain a residue capable of hydrogen bonding at this position, including for example the $\mathrm{L}_{(-8)} \mathrm{N}_{(-2)} \mathrm{I}_{(-1)} \mathrm{N}$ motif of Asn-100 in MYPT1, suggesting that this may be a general property of $\mathrm{FlH}$-mediated ARD hydroxylation. Analyses of the published MYPT1 ARD structure [13] suggest that all three FIH hydroxylation sites are predicted to create a hydrogen bond to the -2 residue (Figure S4). Studies on a synthetic ankyrin-repeat protein have shown that asparaginyl hydroxylation can stabilize the ARD fold [23]. However, whether the three hydroxylated residues in MYPT1 act in synergy to increase the stability of the canonical ARD fold remains to be determined.

In contrast to $1 \mathrm{~KB} \alpha$ and Notch1, which contain two target Asn residues that are differentially hydroxylated $[5,6]$, the three sites of MYPT1 are hydroxylated to approximately the same extent. Although in 293T cells hydroxylation of the overexpressed MYPT1 ARD was at a relatively modest level $(5-10 \%)$, this is likely due to saturation of the endogenous $\mathrm{FIH}$ : coexpression of exogenous $\mathrm{FIH}$ increased hydroxylation at all three sites essentially to completion. In support of this argument, hydroxylation of the two sites observed in endogenous MYPT1 purified from animal tissue was significantly higher than in the overexpressed ARD from tissue culture cells. Importantly however, hydroxylation at these residues was still incomplete. This is consistent with observations made on ARD proteins purified from tissue culture cells [5, 6], and indicates that incomplete hydroxylation of ARDs is likely to be the physiological norm. This could reflect the fact that endogenous $\mathrm{FIH}$ is also limiting in vivo, perhaps due to the ubiquity and abundance of its substrates. 
Finally, we demonstrate that, similar to Notch, MYPT1 effectively inhibits FIH-dependent suppression of HIF-CAD activity. Interestingly, overexpression of several other known ARD substrates of FIH also enhanced the activity of HIF-CAD, although this effect is not universal and different ARD substrates compete to different extents (Figure 6). It is possible that the ability of an ARD to promote HIF-CAD activity depends on its affinity for $\mathrm{FIH}$, and therefore on the extent to which the ARD physically sequesters FIH away from HIF-CAD.

Given that FIH-mediated hydroxylation is likely to be very common among ARD proteins, and that several ARD substrates apparently inhibit HIF-CAD hydroxylation, it seems plausible that collectively ARDs act as a "sink" for FIH. This would explain not only the observations of limiting FIH activity towards HIF-CAD in tissue culture models [14] but also the incomplete hydroxylation of ARDs themselves, due to cross-competition.

\section{Acknowledgments}

We thank Michael Olson (University of Glasgow, UK), Marino Zerial (Max Planck Institute of Molecular Cell Biology and Genetics, Dresden, Germany) and Catherine Bisbal (Institute of Human Genetics, Montpelier, France) for valuable reagents, Michael McDonough (Chemistry Research Laboratory, University of Oxford) for help with the preparation of the structural figures, and Mariola Edelmann and Benedikt Kessler (Central Proteomics Facility, University of Oxford) for assistance with mass spectrometry. This work was funded by the Wellcome Trust and the Medical Research Council UK. 


\section{References}

1 Coleman, M. L. and Ratcliffe, P. J. (2007) Oxygen sensing and hypoxia-induced responses. Essays Biochem. 043, 1-16

2 Lisy, K. and Peet, D. J. (2008) Turn me on: regulating HIF transcriptional activity. Cell Death Differ. 15, 642-649

3 Lando, D., Peet, D. J., Gorman, J. J., Whelan, D. A., Whitelaw, M. L. and Bruick, R. K. (2002) FIH-1 is an asparaginyl hydroxylase enzyme that regulates the transcriptional activity of hypoxia-inducible factor. Genes Dev. 16, 1466-1471

4 Mahon, P. C., Hirota, K. and Semenza, G. L. (2001) FIH-1: a novel protein that interacts with HIF-1 $\alpha$ and VHL to mediate repression of HIF-1 transcriptional activity. Genes Dev. 15, 2675-2686

5 Cockman, M. E., Lancaster, D. E., Stolze, I. P., Hewitson, K. S., McDonough, M. A., Coleman, M. L., Coles, C. H., Yu, X., Hay, R. T., Ley, S. C., Pugh, C. W., Oldham, N. J., Masson, N., Schofield, C. J. and Ratcliffe, P. J. (2006) Posttranslational hydroxylation of ankyrin repeats in IKB proteins by the hypoxia-inducible factor (HIF) asparaginyl hydroxylase, factor inhibiting HIF (FIH). Proc. Natl. Acad. Sci. USA. 103, 14767-14772

6 Coleman, M. L., McDonough, M. A., Hewitson, K. S., Coles, C., Mecinovic, J., Edelmann, M., Cook, K. M., Cockman, M. E., Lancaster, D. E., Kessler, B. M., Oldham, N. J., Ratcliffe, P. J. and Schofield, C. J. (2007) Asparaginyl Hydroxylation of the Notch Ankyrin Repeat Domain by Factor Inhibiting Hypoxia-inducible Factor. J. Biol. Chem. 282, 2402724038

7 Ferguson, J. E., III, Wu, Y., Smith, K., Charles, P., Powers, K., Wang, H. and Patterson, C. (2007) ASB4 Is a Hydroxylation Substrate of FIH and Promotes Vascular Differentiation via an Oxygen-Dependent Mechanism. Mol. Cell. Biol. 27, 6407-6419

8 Cockman, M. E., Webb, J. D., Kramer, H. B., Ratcliffe, P. J. and Kessler, B. M. (2009) Proteomics-based identification of novel Factor Inhibiting HIF $(\mathrm{FIH})$ substrates indicates widespread asparaginyl hydroxylation of ankyrin repeat domain-containing proteins. Mol. Cell. Proteomics. doi: $10.1074 / \mathrm{mcp} . M 800340-M C P 200$

9 Zheng, X., Linke, S., Dias, J. M., Zheng, X., Gradin, K., Wallis, T. P., Hamilton, B. R., Gustafsson, M., Ruas, J. L., Wilkins, S., Bitton, R. L., Brismar, K., Whitelaw, M. L., Pereira, T., Gorman, J. J., Ericson, J., Peet, D. J., Lendahl, U. and Poellinger, L. (2008) Interaction with factor inhibiting HIF-1 defines an additional mode of cross-coupling between the Notch and hypoxia signaling pathways. Proc. Natl. Acad. Sci. USA. 105, 3368-3373

10 Mosavi, L. K., Cammett, T. J., Desrosiers, D. C. and Peng, Z.-Y. (2004) The ankyrin repeat as molecular architecture for protein recognition. Protein Sci. 13, 1435-1448

11 Schultz, J., Milpetz, F., Bork, P. and Ponting, C. P. (1998) SMART, a simple modular architecture research tool: Identification of signaling domains. Proc. Natl. Acad. Sci. USA. 95, 5857-5864

12 Ito, M., Nakano, T., Erdödi, F. and Hartshorne, D. J. (2004) Myosin phosphatase: structure, regulation and function. Mol. Cell. Biochem. 259, 197-209

13 Terrak, M., Kerff, F., Langsetmo, K., Tao, T. and Dominguez, R. (2004) Structural basis of protein phosphatase 1 regulation. Nature. 429, 780-784

14 Stolze, I. P. Tian, Y. M., Appelhoff, R. J., Turley, H., Wykoff, C. C., Gleadle, J. M. and Ratcliffe, P. J. (2004) Genetic analysis of the role of the asparaginyl hydroxylase factor inhibiting hypoxia-inducible factor (HIF) in regulating HIF transcriptional target genes. J. Biol. Chem. 279, 42719-42725

15 Wu, Y., Erdődi, F., Murányi, A., Nullmeyer, K. D., Lynch, R. M. and Hartshorne, D. J. (2003) Myosin phosphatase and myosin phosphorylation in differentiating C2C12 cells. J. Muscle Res. Cell Motil. 24, 499-511

16 Kinter, M. and Sherman, N. E. (2000) Protein sequencing and identification using tandem mass spectrometry. John Wiley \& Sons Inc., New York

17 Perkins, D. N., Pappin, D. J. C., Creasy, D. M. and Cottrell, J. S. (1999) Probabilitybased protein identification by searching sequence databases using mass spectrometry data. Electrophoresis. 20, 3551-3567

18 Shimizu, H., Ito, M., Miyahara, M., Ichikawa, K., Okubo, S., Konishi, T., Naka, M., Tanaka, T., Hirano, K., Hartshorne, D. J. and Nakano, T. (1994) Characterization of the myosin-binding subunit of smooth muscle myosin phosphatase. J. Biol. Chem. 269, 3040730411 
19 Alessi, D., MacDougall, L. K., Sola, M. M., Ikebe, M. and Cohen, P. (1992) The control of protein phosphatase-1 by targetting subunits. Eur. J. Biochem. 210, 1023-1035

20 Hirano, K., Phan, B. C. and Hartshorne, D. J. (1997) Interactions of the Subunits of Smooth Muscle Myosin Phosphatase. J. Biol. Chem. 272, 3683-3688

21 Tanaka, J., Ito, M., Feng, J., Ichikawa, K., Hamaguchi, T., Nakamura, M., Hartshorne, D. J. and Nakano, T. (1998) Interaction of Myosin Phosphatase Target Subunit 1 with the Catalytic Subunit of Type 1 Protein Phosphatase. Biochemistry. 37, 16697-16703

22 Tóth, A., Kiss, E., Herberg, F. W., Gergely, P., Hartshorne, D. J. and Erdődi, F. (2000) Study of the subunit interactions in myosin phosphatase by surface plasmon resonance. Eur. J. Biochem. 267, 1687-1697

23 Kelly, L., McDonough, M. A., Coleman, M. L., Ratcliffe, P. J. and Schofield, C. J. (2009) Asparagine beta-hydroxylation stabilizes the ankyrin repeat domain fold. Mol. Biosyst. $5,52-58$ 


\section{Figure legends}

\section{Figure 1}

MYPT1 is a potential FIH substrate

Alignment of known FIH substrates with four putative hydroxylation sites in human MYPT1: Asn-67, Asn-100, Asn-226 and Asn-288. Arrow indicates target asparagine residues. Sequences were aligned using ClustalW and shaded using BoxShade.

\section{Figure 2}

The ARD of MYPT1 is hydroxylated in vivo

Representative MS/MS spectra showing the tryptic fragment of human MYPT1 containing Asn-226 (LLIQAGYDVNIK) in unoxidized and hydroxylated states. Spectrum A shows the unoxidized peptide, whilst spectrum $\mathbf{B}$ shows the hydroxylated peptide. In $\mathbf{B}$ an additional mass of $16 \mathrm{Da}$ is observed in the b ion series from b10 onwards, and in all the observed $y$ ions, indicating hydroxylation on Asn-226.

\section{Figure 3}

FIH is necessary and sufficient for hydroxylation of the MYPT1 ARD

(A) 293T cells were treated with siRNAs against FIH (FIHi) or a control sequence (dHIFi). Following siRNA treatment, cells were transfected with V5-tagged MYPT1 ARD and the indicated FIH construct: pcDNA3 vector (EV), wild-type FIH or hydroxylase-inactive D201A FIH. MYPT1 ARD was immunopurified, digested and analysed by LC-MS to quantitate hydroxylation. ${ }^{* * *}$ denotes $p<0.001$ relative to $\mathrm{dHIFi/EV}$ control. Due to sample loss the value for Asn-226 with D201A overexpression is presented as the mean of two independent experiments.

(B) Levels of FIH in the cell extracts were determined by immunoblotting; a representative immunoblot is shown.

\section{Figure 4}

Endogenous MYPT1 is hydroxylated

(A) Extracted ion chromatograms of $\mathrm{m} / \mathrm{z}=1012.3$ (solid line) and 1017.4 (broken line), corresponding to the unhydroxylated and hydroxylated forms of the tryptic peptide containing Asn-67 (GADINYANVDGLTALHQACIDDNVDMVK). The $\mathrm{m} / \mathrm{z}$ difference of 5.1 Da between the triply-charged ions corresponds to a mass difference of 16 Da between the uncharged peptides within the mass resolution of the instrument.

(B) Extracted ion chromatograms of $\mathrm{m} / \mathrm{z}=593.7$ (solid line) and 601.8 (broken line), corresponding to the unhydroxylated and hydroxylated forms of the GluC peptide containing Asn-100 (NGANINQPDNE). The $\mathrm{m} / \mathrm{z}$ difference of 8.1 Da between the doubly-charged ions corresponds to a mass difference of $16 \mathrm{Da}$ between the uncharged peptides within the mass resolution of the instrument.

\section{Figure 5}

FIH does not affect the activity of myosin phosphatase, nor the interaction between MYPT1 and PPIc $\delta$

(A) 293T cells were transfected with pcDNA3 vector (EV), wild-type FIH, D201A FIH, control siRNA (dHIFi) or siRNA targeting $\mathrm{FIH}(\mathrm{FIHi})$. After immunoprecipitation of endogenous MYPT1, immunoprecipitates and inputs were analysed for MYPT1, PP1c $\delta, M L C, F I H$ and $\beta-$ tubulin by immunoblotting.

(B) 293T HeLa and MCF7 cells were transfected as in A. Extracts were examined for MYPT1, pMLC (Ser-19), pMLC (Thr-18/Ser-19), MLC, FIH and $\beta$-tubulin by immunoblotting.

Figure 6

MYPT1 and other ARD-containing proteins can influence HIF transcriptional activity through competition for $\mathrm{FIH}$

(A) HeLa cells were transfected with UAS Luciferase, HIF-CAD-GAL DBD, the indicated 3xFLAG-tagged ARD protein (mN1 ICD: mouse Notch1 intracellular domain; TNKS1: Tankyrase 1 ) and $\beta$-galactosidase as a transfection control. $48 \mathrm{~h}$ post-transfection, cells were harvested and analysed in triplicate for luciferase and $\beta$-galactosidase activity. ${ }^{* *}$ denotes $p<$ 0.01 relative to EV control sample. 
(B) Cell extracts were analysed by anti-FLAG immunoblotting to confirm similar expression of the ARD proteins. 
Biochemical Journal Immediate Publication. Published on 27 Feb 2009 as manuscript BJ20081905

\section{Figures}

\section{Figure 1}

HIF 1 a N 803

p105- 678

$\mathrm{IkBa}-\mathrm{N} 210$

$\mathrm{I} \mathrm{kBa}{ }^{-} \mathrm{N} 244$

$\mathrm{mN} 1 \overline{\mathrm{N}} 1945$

$\mathrm{mN} 1{ }^{-} \mathrm{N} 2012$

ASB $\overline{4}$ N 246

MYPT'

MYPT1 N100

MYPT1-N226

MYPT1_N288

DESGLPQLT SYDCEVNAPIQ - GSRNLTQGEEL SL PCLLLLVAAGADVNAQEQKS GRTALHLAV YLGIVELLVSLGADVNAQE P CNGR TALHLAV NPDLVSLLLKCGADVNRVTY - QGYSPYQLTWG RS DAAKRLLEASADANI QDN - MGR T P LHAAVS VEGMLEDL IN S HADVNA VDD - LGK SALHWAAA HHLVCRMLLDYKAEVNARDD - DFK SPLHKAAW DTDEVLKLLHRGADINYANV - DGLTALHOACI NVDMVKFLVENGANINQPDN - EGW I PLHAAAS YTEVLKLIIQAGYDVNIKDY - DGWTPLHAAAH DEDILGYLEELQKKQNLLHS - EKRDKKSPLIE

Licenced copy. Copying is not permitted, except with prior permission and as allowed by law. (C) 2009 The Authors Journal compilation (c) 2009 Portland Press Limited 


\section{Figure 2}

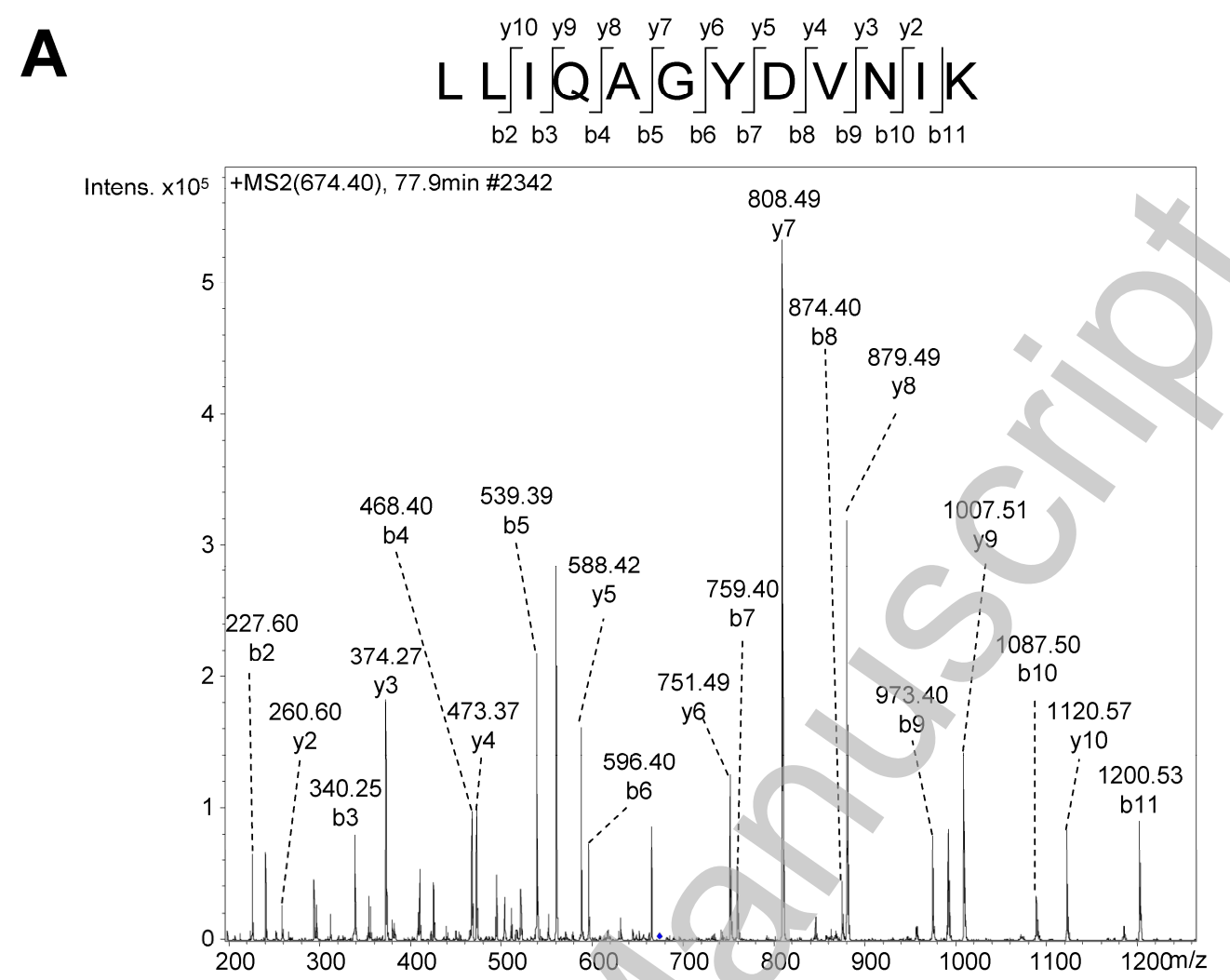

B

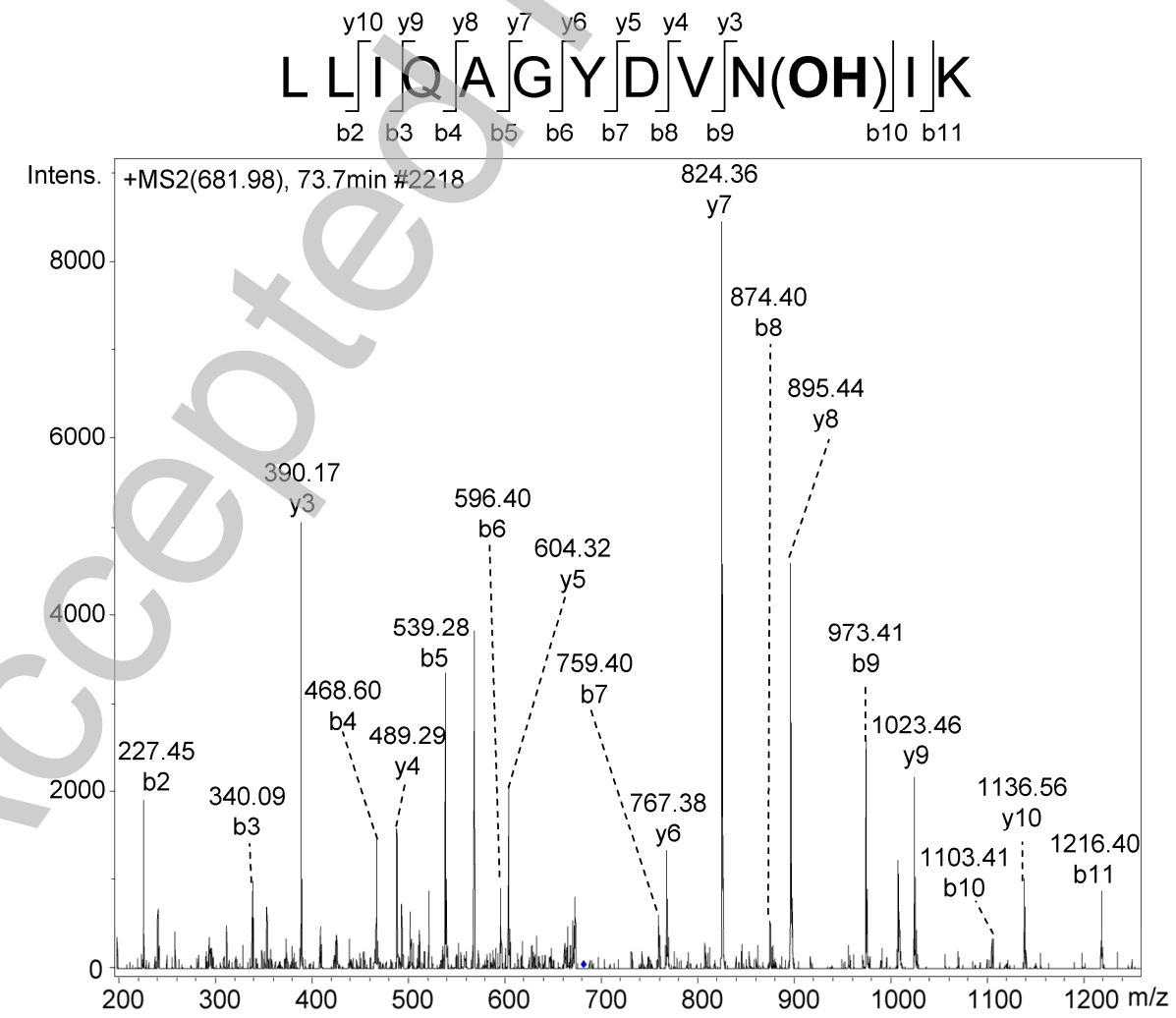




\section{Figure 3}

A

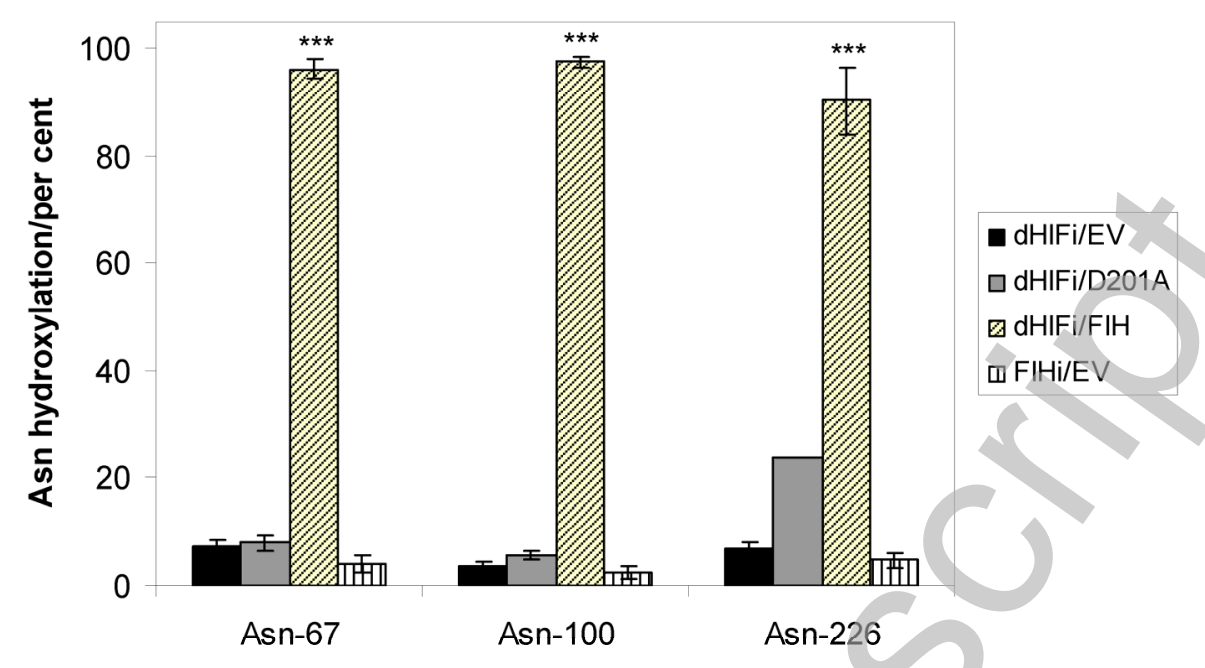

B

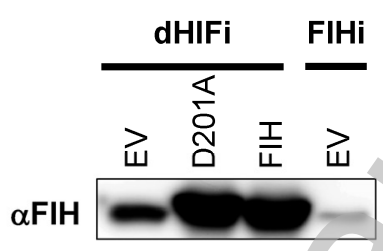




\section{Figure 4}

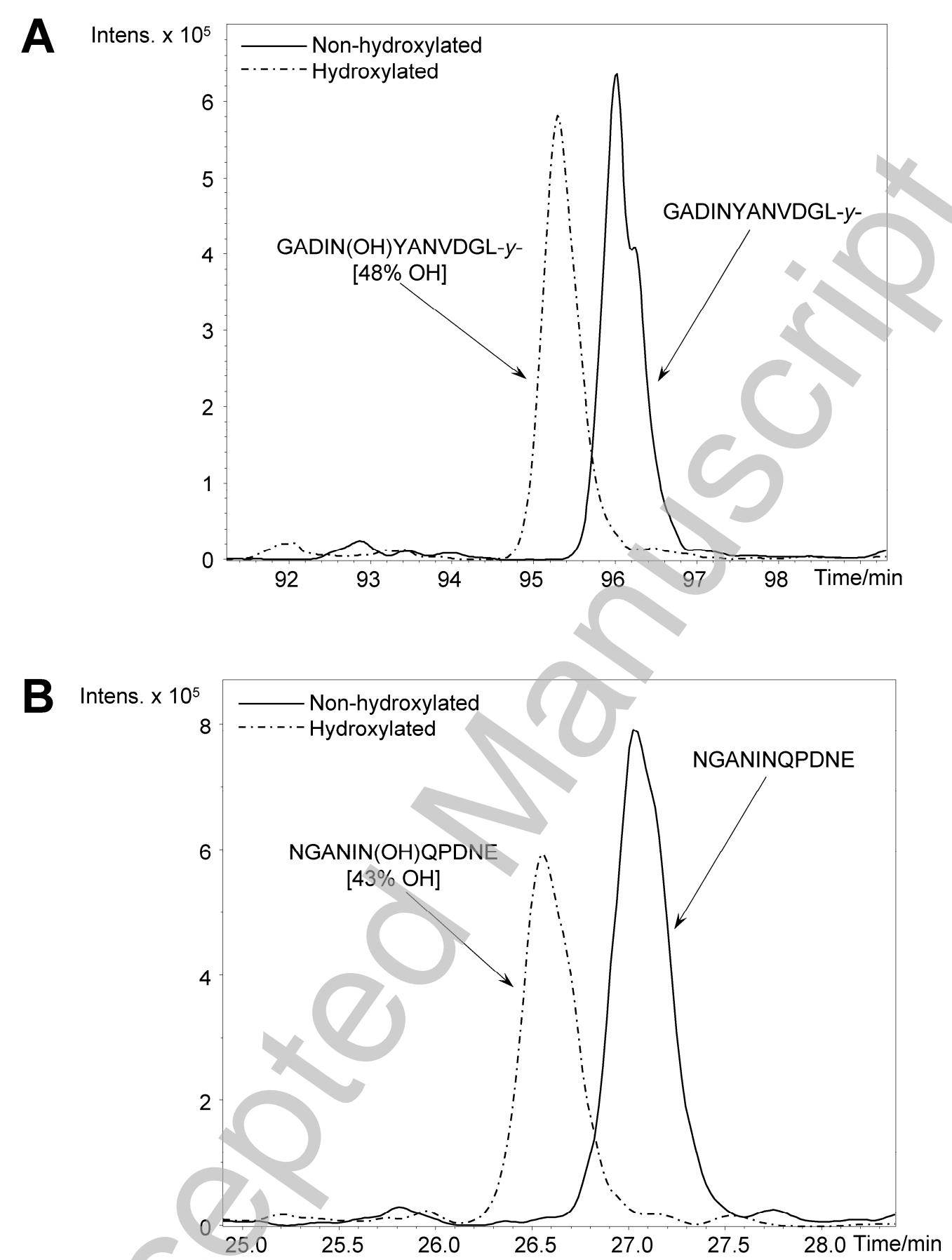


Figure 5

A

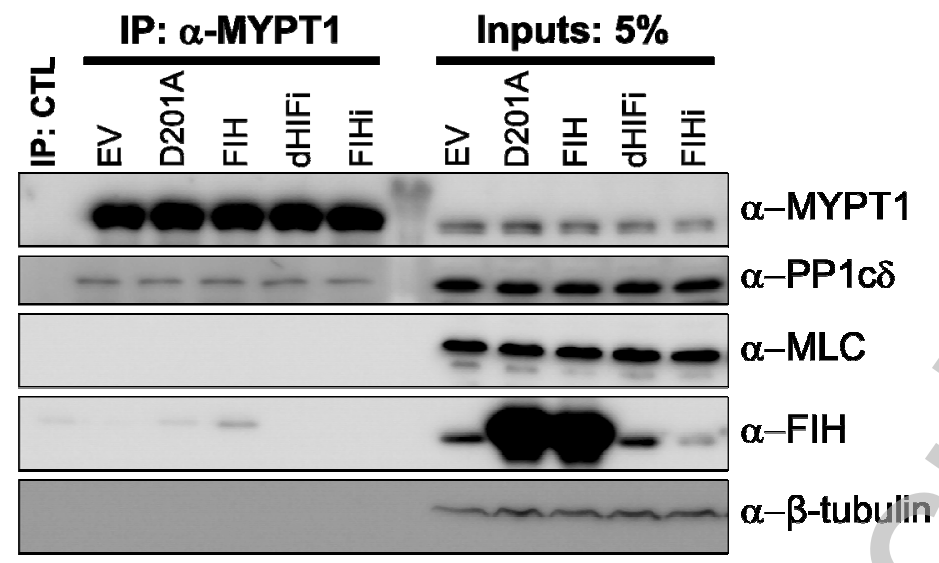

B
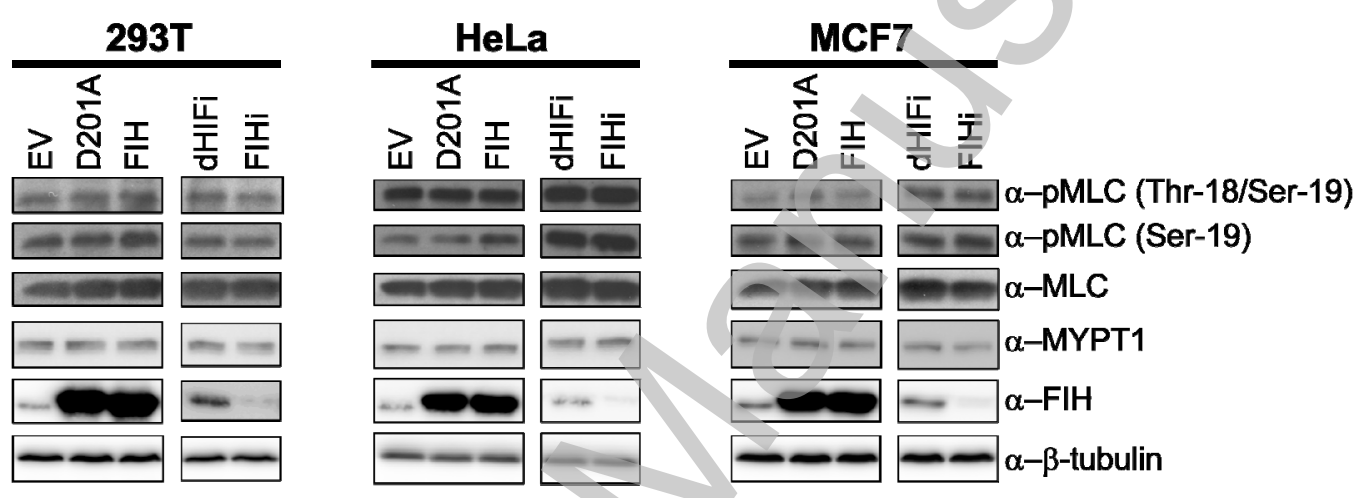

$\square \alpha-$ pMLC (Thr-18/Ser-19)

$\Longrightarrow \alpha-p M L C$ (Ser-19)
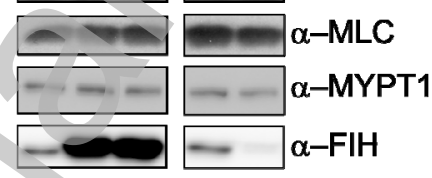

$-\infty \alpha-\beta$-tubulin 


\section{Figure 6}

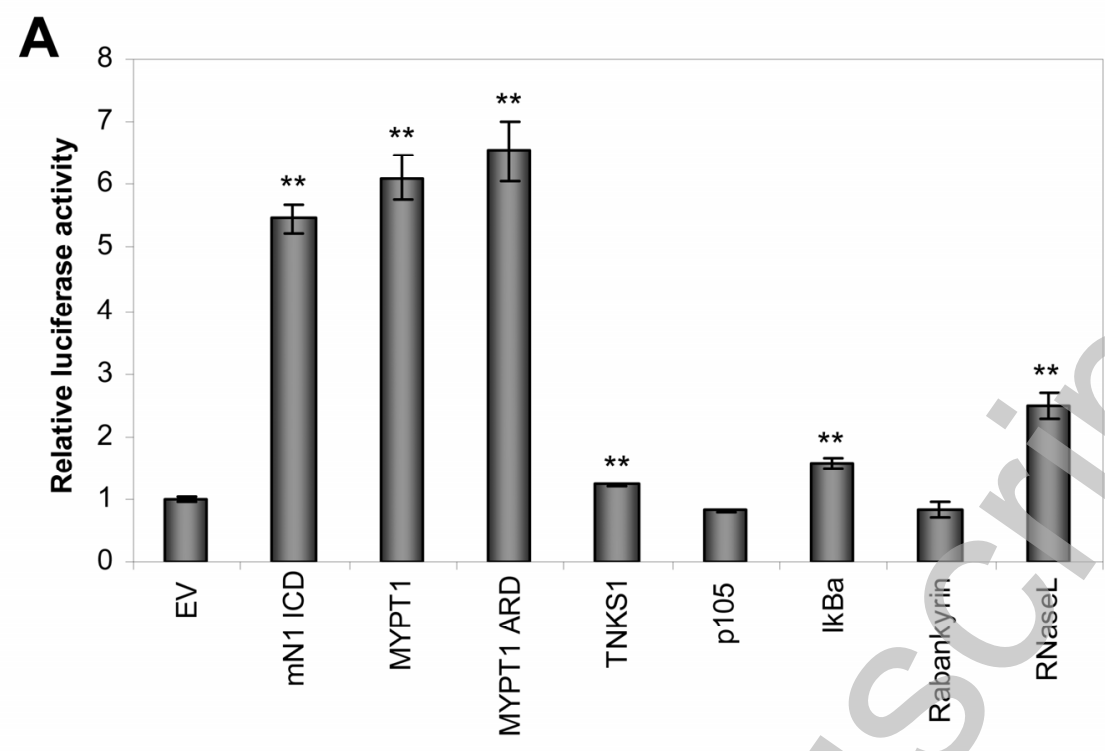

B

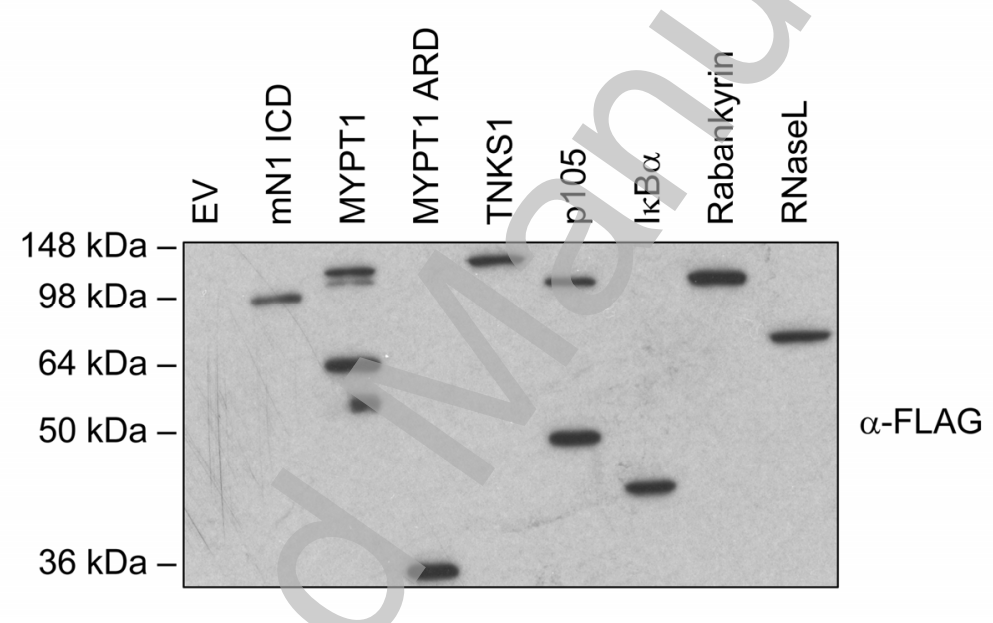

